\title{
DIMENSIONAMENTO E SIMULAÇÃO DE UMA MICRORREDE UTILIZANDO GERAÇÃO CONJUNTA EÓLICA/SOLAR APLICADA EM UM CONDOMÍNIO RESIDENCIAL NA CIDADE DE JANAÚBA/MG ${ }^{1}$
}

\author{
Natália Rodrigues de Melo ${ }^{2}$ \\ Rafael Vinícius Tayette de Nobrega ${ }^{3}$
}

\begin{abstract}
RESUMO
Uma microrrede pode ser definida como um sistema de geração de energia de pequeno porte contendo unidades geradoras distribuídas que, normalmente, utilizam diferentes fontes de energia para atender um determinado número de unidades consumidoras. No Brasil, existem alguns fatores que têm contribuído para uma maior inserção das microrredes no sistema elétrico, podendo-se destacar a crise hídrica atual, a existência de centros de consumo afastados do sistema de transmissão e/ou distribuição, questões ambientais e elevado potencial das fontes renováveis no País. Desse modo, este trabalho teve como objetivo dimensionar e simular uma microrrede utilizando a geração eólica/solar, juntamente com um sistema de armazenamento de energia, para atender a demanda de um condomínio com trinta e seis residências, na cidade de Janaúba/MG, que apresenta alto potencial de geração eólica e solar. A simulação do projeto foi realizada através do software HOMER, que é uma ferramenta computacional open source especializada na análise de microrredes. De posse dos valores de irradiância solar e velocidade do vento na cidade de Janaúba/MG, pôde-se concluir que a região apresenta potencial eólico superior ao solar. Assim, o sistema foi dimensionado satisfatoriamente, sendo $66,5 \%$ de sua demanda total fornecidos pelo sistema eólico, e os $33,5 \%$ restantes, pelo sistema solar.
\end{abstract}

Palavras-chave: Microrredes. Fontes renováveis. Software HOMER.

\section{INTRODUÇÃO}

A matriz energética brasileira é considerada uma das melhores do mundo devido ao alto potencial de recursos renováveis disponíveis no País, sendo que $87 \%$ da matriz brasileira

\footnotetext{
${ }^{1}$ Como citar este artigo:

MELO, N. R. de; NOBREGA, R. V. T. de. Dimensionamento e simulação de uma microrrede utilizando geração conjunta eólica/solar aplicada em um condomínio residencial na cidade de Janaúba/MG. ForScience: revista científica do IFMG, Formiga, v. 5, n. 3, e00283, jul./dez. 2017.
}

${ }^{2}$ Mestranda em Engenharia Elétrica pela Universidade Federal de São João Del Rei. (http://lattes.cnpq.br/1790350244865693). E-mail: nathyrmelo@ yahoo.com.br.

${ }_{3}^{3}$ Doutorando em Ciências pela Escola de Engenharia de São Carlos - Universidade de São Paulo (USP) e, atualmente é professor efetivo do Instituto Federal de Educação, Ciências e Tecnologia de Minas Gerais Campus Formiga. (http://lattes.cnpq.br/7492232498866714). E-mail: rafael.nobrega@ifmg.edu.br.

ForSci.: r. cient. IFMG, Formiga, v. 5, n. 3, e00283, jul./dez. 2017. 
são referentes às fontes renováveis, enquanto apenas $13 \%$ da matriz energética mundial possuem participação em energias renováveis (GOMES, 2013).

Dentre as fontes renováveis disponíveis, é importante destacar a dependência que o País possui da geração hidráulica, correspondente a aproximadamente $62,5 \%$ da geração total (MINISTÉRIO DE MINAS E ENERGIA, 2016). Entretanto, nos últimos anos, o volume de chuvas no Brasil tem diminuído drasticamente, comprometendo a geração de energia proveniente das hidrelétricas.

Assim, a inserção das microrredes no cenário energético brasileiro resultaria em uma menor utilização das hidrelétricas e termelétricas, diminuindo assim preocupações relacionadas ao volume dos reservatórios devido a mudanças climáticas. Podem-se considerar, também, os benefícios que a utilização das microrredes trariam ao meio ambiente, diretamente afetado pelo combustível utilizado nas termelétricas, considerado altamente poluente. Sendo assim, as microrredes aproveitariam o potencial de fontes renováveis que o País possui, sem contribuir para uma maior degradação do meio ambiente.

Dessa forma, o uso das microrredes apresenta diversos benefícios sendo importante a realização de estudos nessa área para que essa tecnologia seja cada vez mais difundida. Com isso, o objetivo deste artigo é dimensionar e simular um modelo de microrrede contendo as fontes de energia renováveis solar e eólica, e um sistema de armazenamento de energia utilizando baterias. O projeto propõe que a microrrede opere desconectada da rede elétrica convencional (sistema off-grid), onde o dimensionamento foi realizado considerando como carga principal um condomínio residencial com trinta e seis casas, localizado na cidade de Janaúba/MG. A escolha dessa cidade deve-se ao seu alto potencial de geração eólica e solar.

Este artigo é organizado em cinco seções, sendo que a Seção 2 inclui conceitos sobre a geração distribuída, microrredes, microfontes e as suas características. A Seção 3 apresenta a metodologia desenvolvida no trabalho. Os resultados obtidos e as suas discussões são descritos na Seção 4. Por fim, na Seção 5, são apresentadas as conclusões.

\section{REFERENCIAL TEÓRICO}

\subsection{Geração distribuída}


Perdas de energia, custos ambientais, distâncias dos grandes potenciais hidrelétricos e a necessidade de geração limpa de energia aumentam os incentivos para a utilização de fontes alternativas de energia e próximas dos consumidores (CRUZ, 2013).

Diante dessas circunstâncias, surge o conceito de Geração Distribuída (GD), que é a forma de geração de energia junto ou próxima dos consumidores, que independe da potência gerada, da tecnologia utilizada e dos tipos de fontes de energia envolvidas (INEE, 2016). Para esse tipo de produção, normalmente os consumidores utilizam fontes de energia renováveis, como: eólica, solar, hidráulica e biomassa.

A GD possui vantagens como, por exemplo, diminuição de custos com distribuição e transmissão, queda nas perdas do sistema elétrico, aproveitamento de recursos renováveis locais, confiabilidade, dentre outras. Existem duas modalidades quando se fala em GD: a microgeração e a minigeração. $\mathrm{O}$ critério de diferenciação entre elas refere-se à potência instalada, sendo que, para a microgeração, este valor deve ser menor ou igual a $100 \mathrm{~kW}$, e, para a minigeração, a potência instalada deve ser superior a $100 \mathrm{~kW}$ e menor ou igual a $1 \mathrm{MW}$ (ANEEL, 2014). A resolução normativa ${ }^{\circ}$ 482, de 2012, da ANEEL, apresenta as condições gerais para o acesso de microgeração e minigeração distribuída aos sistemas de distribuição de energia elétrica e dá instruções relacionadas a esse tipo de sistema. A mais recente modificação dessa resolução normativa é a de $n^{\circ}$ 687, publicada em 2015 (ANEEL, 2015).

\subsubsection{Microrredes}

Uma microrrede pode ser definida como uma aplicação do conceito de GD, sendo caracterizada pela sua funcionalidade, e não pelo tamanho. As microrredes envolvem a interconexão de pequenos geradores e cargas em uma rede local, podendo funcionar conectadas à rede principal de distribuição de energia ou operar isoladamente. Elas consistem numa variedade de componentes, incluindo geradores distribuídos, elementos armazenadores de energia e cargas (SU; WANG, 2012).

As microrredes podem ser úteis para suprir cargas elétricas onde há dificuldades de acesso da rede de distribuição convencional, como em zonas rurais afastadas, áreas de florestas ou ilhas fluviais. Além disso, também podem ser projetadas para pequenas comunidades, como conjuntos habitacionais, comunidades acadêmicas (universidades e 
escolas), áreas comerciais e locais que exigem fornecimento ininterrupto de energia elétrica, podendo-se destacar os hospitais e os sistemas prisionais.

As microfontes que compõem uma microrrede são pequenas unidades geradoras de centenas de $\mathrm{kW}$ ou menos. As principais microfontes utilizadas são módulos fotovoltaicos, geradores eólicos, microturbinas a gás, células a combustível, incluindo também as microcentrais hidrelétricas (SILVA, 2011). A Figura 1 mostra as partes que constituem uma microrrede, englobando as microfontes, os sistemas de armazenamento de energia, utilizando baterias e sistema de controle; e as cargas.

Um dos objetivos principais das microrredes é combinar fontes de energia não convencionais ou renováveis de baixa emissão de carbono em sistemas de geração de alta eficiência (PASSOS, 2014).

A escolha sobre qual microfonte utilizar depende da disponibilidade do recurso na área de implementação da microrrede. As principais microfontes utilizadas para geração distribuída são: sistemas de energia e calor combinados (CHP), microturbinas, células de combustível, geradores eólicos, geradores fotovoltaicos e pequenas centrais hidrelétricas $(\mathrm{PCH})$. No presente trabalho, foram utilizadas apenas as microfontes eólica e solar.

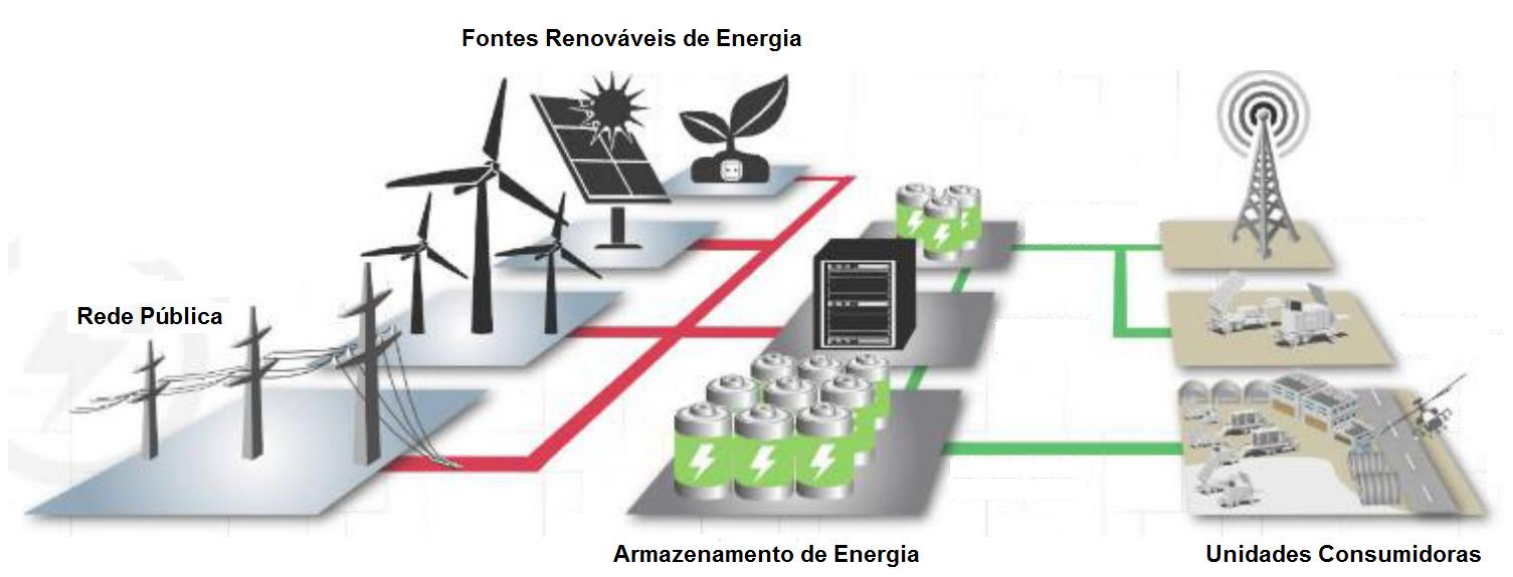

Figura 1 - Representação esquemática das partes constituintes de uma microrrede Fonte: Adaptada de Raytheon Company (2015).

\subsubsection{Dispositivos armazenadores de energia}

O armazenamento de energia elétrica nas microrredes é de extrema importância, devido à característica intermitente das fontes de energia renováveis utilizadas. $\mathrm{O}$ uso de um sistema de armazenamento possibilita que a energia seja aproveitada posteriormente, 
aumentando assim a eficiência do sistema (OUDALO.; BUEHLER; CHARTOUNI, 2006). Atualmente, os dispositivos armazenadores de energia mais utilizados são as baterias, os discos de inércia (flywheels) e os supercapacitores.

Entre todas as tecnologias disponíveis para utilização em microrredes, os bancos de baterias são as mais utilizadas para armazenamento de energia. Com relação às desvantagens relacionadas a esse tipo de sistema, podem-se citar seu alto custo e a necessidade de um espaço disponível para alocação das baterias, que são muito espaçosas.

\subsubsection{Sistemas on-grid e off-grid}

Uma microrrede pode operar conectada ou não à rede elétrica convencional. Os sistemas que operam conectados são denominados on-grid, e aqueles que operam isoladamente são conhecidos como sistemas off-grid.

A configuração on-grid permite que a carga seja atendida pela geração das fontes renováveis quando esse sistema possui condições favoráveis de funcionamento, por exemplo, irradiância necessária e valores adequados de velocidade do vento. Quando é detectado um excesso na produção de energia, este excedente pode ser enviado à rede, e o consumidor é recompensado por isso. Além disso, quando as fontes renováveis de energia não estão disponíveis, a rede elétrica fornecerá energia suficiente para atender à demanda.

Os sistemas off-grid necessitam de baterias para fornecer energia em períodos nos quais a geração por meio das fontes renováveis não é suficiente para atender a carga, assegurando asssim um fornecimento contínuo. Os bancos de baterias normalmente precisam ser substituídos após 10 anos de uso, além de possuírem alto custo e reduzirem a eficiência total do sistema. Normalmente, esse tipo de sistema é utilizado em áreas que não podem ser atendidas por uma rede elétrica; caso contrário, a independência é a principal razão pela qual o consumidor opta por um sistema off-grid (SINGH et al., 2016).

\subsubsection{O sistema Ingecon Hybrid MS}

O sistema Ingecon Hybrid MS foi implementado para operar desconectado da rede elétrica e fornecer tensão AC utilizando energia proveniente das fontes renováveis solar e eólica. Além disso, utiliza baterias como meio de armazenamento e pode ser conectado a um 
gerador de segurança (backup) capaz de fornecer energia para a carga a qualquer momento. Este gerador somente é utilizado quando a energia proveniente das fontes renováveis e/ou das baterias não for suficiente para atender a carga, e pode ser um gerador a diesel ou até mesmo a rede elétrica convencional. Sendo assim, o sistema híbrido pode funcionar conectado ou não à rede elétrica. A Figura 2 mostra a configuração do sistema Ingecon Hybrid MS com seus respectivos blocos de entrada e saída.

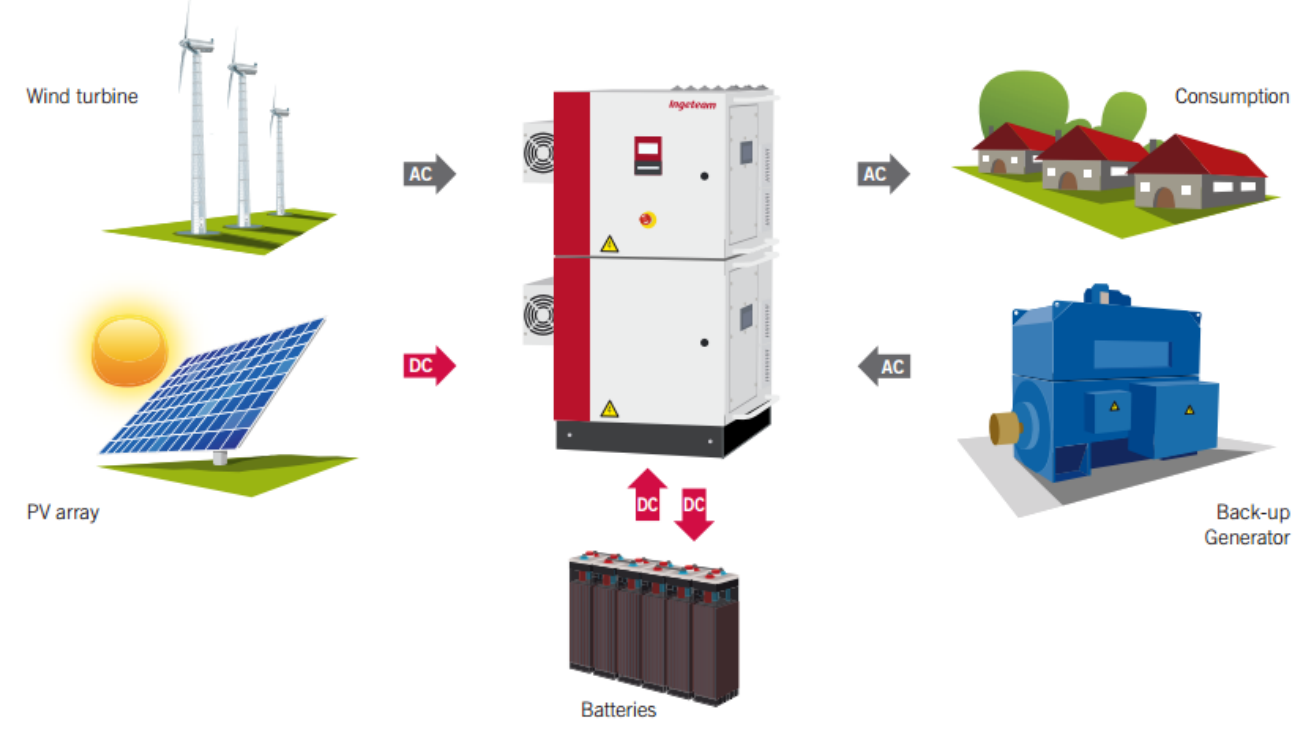

Figura 2 - O sistema Ingecon Hybrid MS indicando suas entradas e saídas Fonte: Ingeteam (2013).

\subsubsection{Potencial eólico e solar de Minas Gerais}

A escolha da região de Minas Gerais para a realização do projeto proposto deveu-se a verificação das áreas mais promissoras para a geração conjunta de energia eólica e solar.

De acordo com Companhia Energética de Minas Gerais (2012), os locais favoráveis para implantação de sistemas fotovoltaicos planos devem possuir radiação solar maior que 2.000 $\mathrm{kWh} / \mathrm{m}^{2}$, ou seja, $5,5 \mathrm{kWh} / \mathrm{m}^{2}$ por dia (valor médio diário anual). Como pode ser visualizado na Figura 3, mais da metade do estado de Minas Gerais (todo o lado ocidental) possui radiação solar global média anual no intervalo entre 5,5 e 6,5 kWh $/ \mathrm{m}^{2}$.dia. Assim, com base nessas informações, considerou-se que o estado dispõe de vastas regiões que preenchem tais requisitos. 


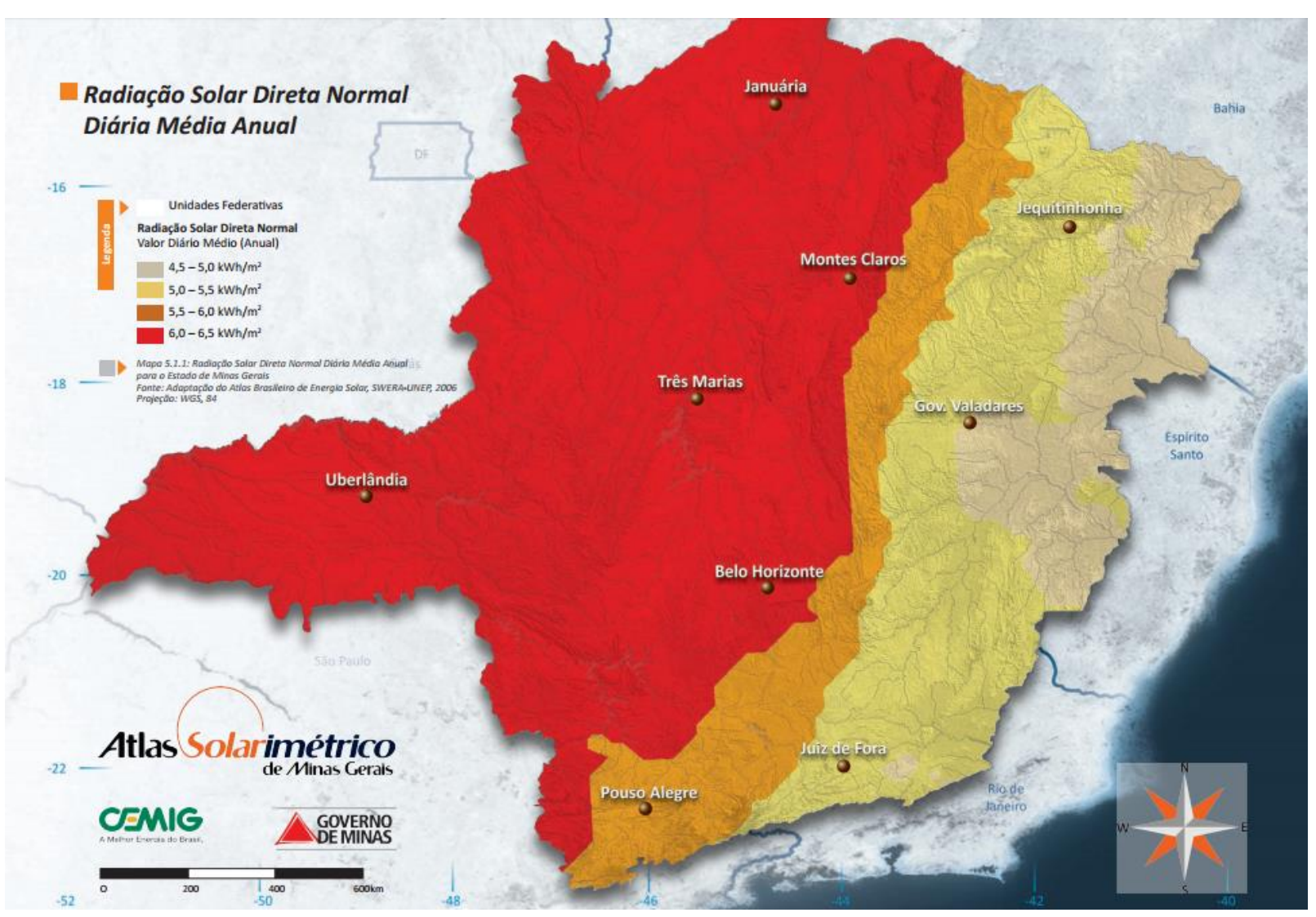

Figura 3 - Radiação solar direta normal diária média anual para o estado de Minas Gerais Fonte: Companhia Energética de Minas Gerais (2012).

Os limiares mínimos de atratividade para investimento em geração eólica dependem dos contextos econômicos e institucionais de cada país, variando em termos de velocidades médias anuais dos ventos entre 5,5 e 7,0 m/s. Entretanto, estima-se que aquelas acima de 6,0 $\mathrm{m} / \mathrm{s}$ já apontam condições favoráveis para o investimento em usinas eólicas (COMPANHIA ENERGÉTICA DE MINAS GERAIS, 2010). As áreas com maior potencial eólico no estado podem ser visualizadas na Figura 4. 


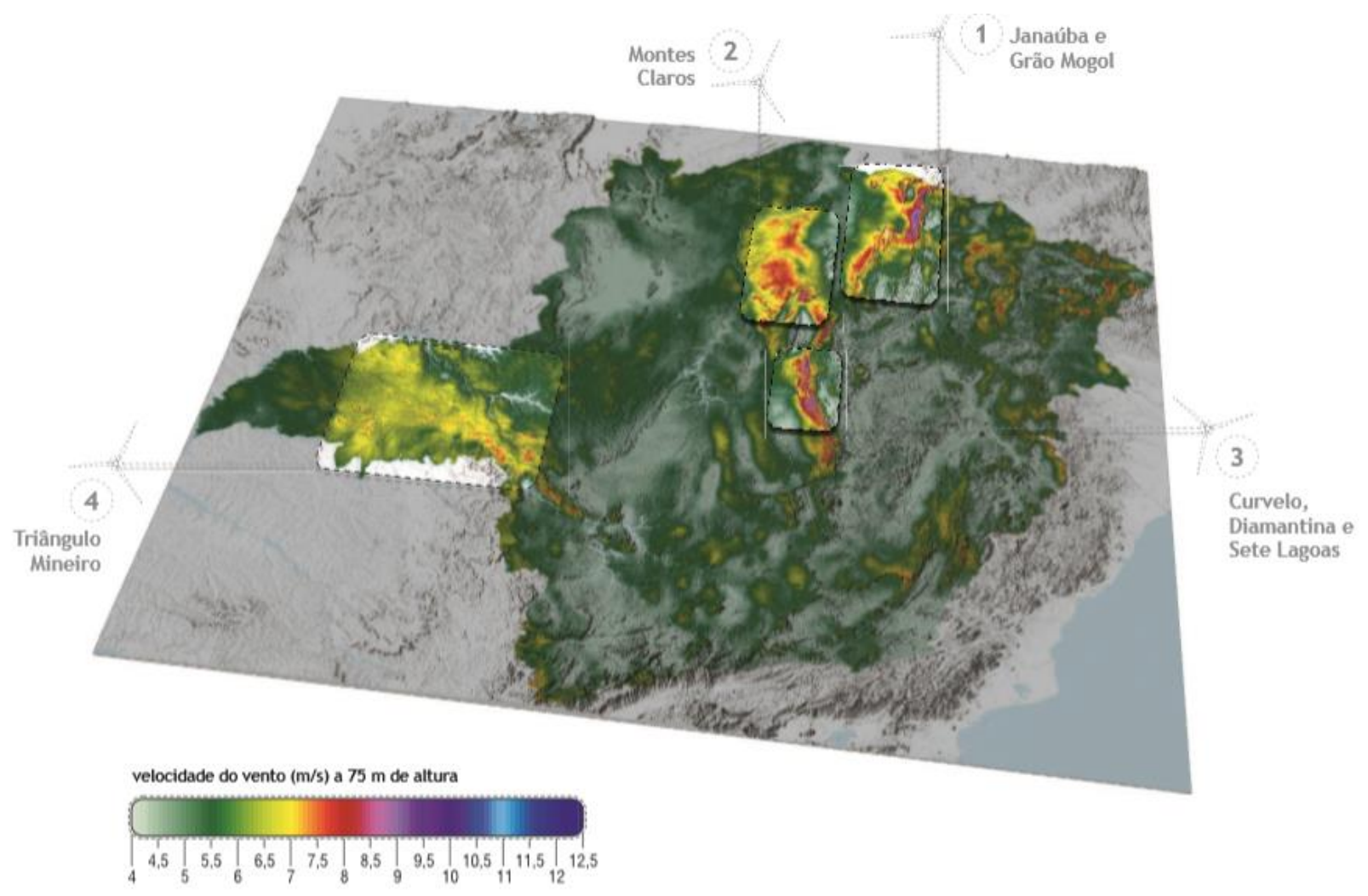

Figura 4 - Áreas mais promissoras para geração eólica em Minas Gerais.

Fonte: Companhia Energética de Minas Gerais (2010).

Com base nas informações apresentadas, a cidade de Janaúba/MG foi escolhida como base para o projeto de microrrede por apresentar resultados satisfatórios de potencial eólico e solar. A região de Janaúba é a mais dotada de radiação solar no estado de Minas Gerais, com valores anuais de 2.200 a $2.400 \mathrm{kWh} / \mathrm{m}^{2}$, podendo atingir, no verão, 2.500 a $2.700 \mathrm{kWh} / \mathrm{m}^{2}$, e possui também alta predominância de ventos, alcançando valores superiores a 7,5 m/s a $75 \mathrm{~m}$ de altura. Além disso, a maior parte do território tem baixa aptidão agrícola, baixa declividade e ausência de unidades de conservação, o que favorece positivamente a geração de energia eólica (COMPANHIA ENERGÉTICA DE MINAS GERAIS, 2012). Assim, na próxima seção, é apresentada a metodologia desenvolvida neste artigo.

\section{METODOLOGIA DESENVOLVIDA}

Neste trabalho, são apresentados os cálculos pertinentes à microrrede. Posteriormente, o sistema foi dimensionado pelo software HOMER, ferramenta computacional open source especializada em projetos de microrredes. 


\subsection{O software HOMER}

O software HOMER é utilizado para implementar e analisar sistemas híbridos de potência que contêm diferentes elementos, como turbinas eólicas, painéis fotovoltaicos, geradores hidráulicos, baterias, inversores, dentre outros. O programa possibilita que tanto sistemas conectados à rede elétrica quanto sistemas que operam isoladamente sejam simulados (OPENEI, 2016). A Figura 5 mostra um fluxograma contendo as etapas de funcionamento do HOMER.

Inicialmente, devem-se definir algumas variáveis de entrada, como informações sobre a carga, dimensionamento dos componentes e seus respectivos custos, disponibilidade dos recursos energéticos e dados utilizados na otimização do sistema. O HOMER usa estas entradas para simular diferentes configurações do sistema, podendo alterar também a combinação de componentes, sendo que os modelos obtidos são relacionados conforme o custo presente líquido (Net Present Cost - NPC) (NATIONAL RENEWABLE ENERGY LABORATORY, 2011).

Posteriormente, é realizada a simulação a fim de verificar se a demanda necessária para atender a carga é atingida. Caso esta condição seja satisfeita, os custos e a produção de energia são avaliados, e o sistema que possui o melhor custo-benefício é escolhido. Caso contrário, os componentes utilizados são redimensionados e inicia-se novamente o processo de simulação.

Sendo assim, o software HOMER foi utilizado para dimensionar e simular o sistema proposto neste artigo. A Figura 6 mostra o sistema construído, composto por painéis solares, turbinas eólicas, baterias, inversor e carga. $\mathrm{O}$ inversor foi utilizado para desempenhar as funções pertinentes ao sistema Ingecon Hybrid MS. 


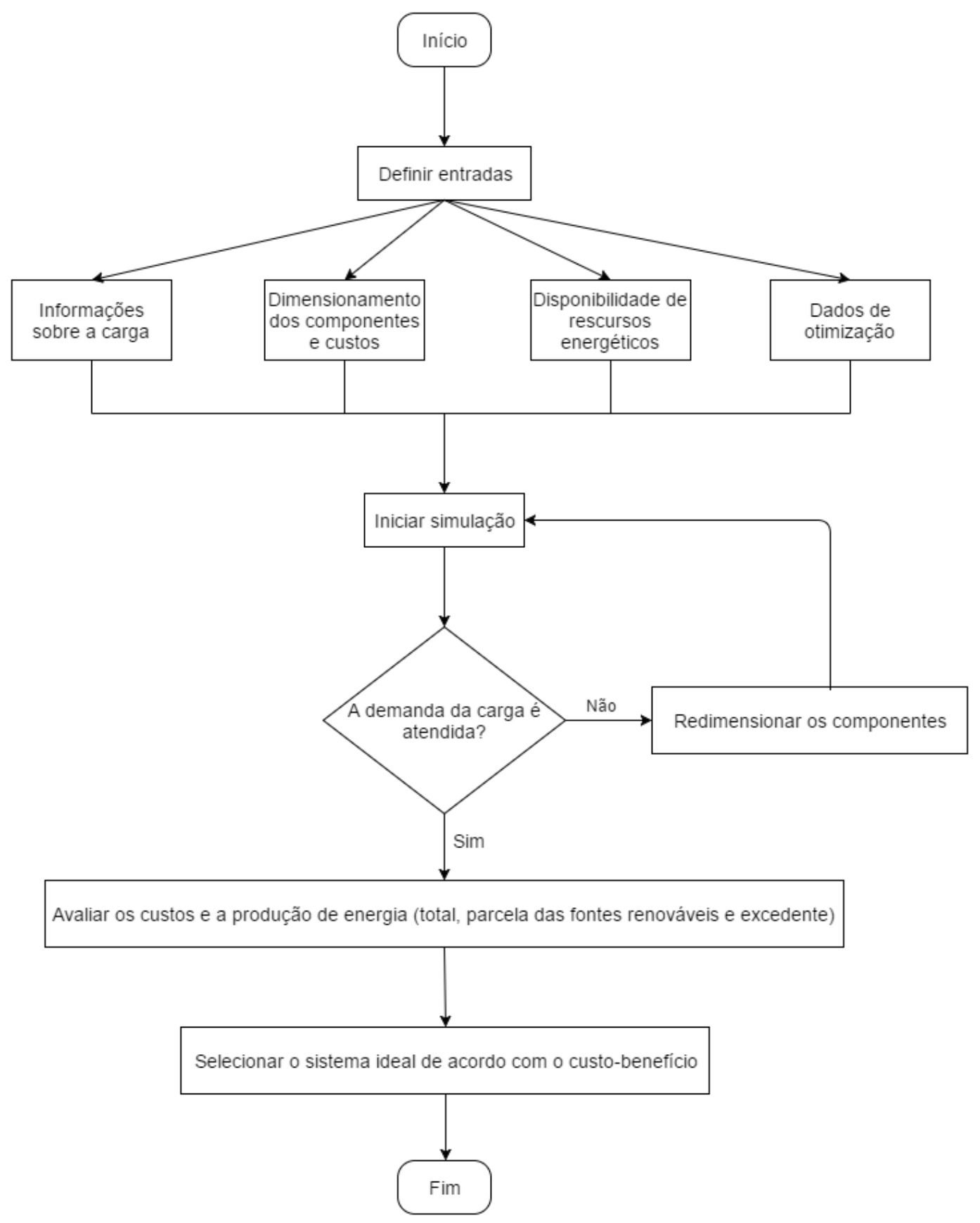

Figura 5 - Fluxograma mostrando o funcionamento do software HOMER Fonte: Dos autores (2016).

Na Figura 6, "G3” representa a turbina eólica; "Electric Load” é referente à carga do condomínio; "Converter" simboliza o inversor; "PV" representa o painel fotovoltaico; e, por fim, "1 kWh LA" é a simbologia utilizada para a bateria. 


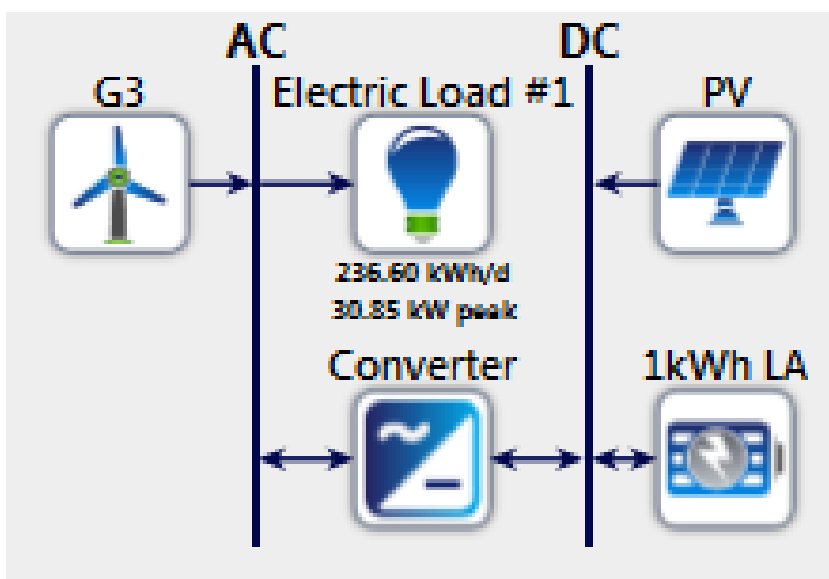

Figura 6 - Arquitetura de microrrede criada no software HOMER Fonte: Homer Energy (2016)

\subsubsection{Variáveis de entrada do software HOMER}

Algumas informações devem ser fornecidas ao programa antes que a análise do sistema seja iniciada. As variáveis de entrada do software para o trabalho em questão foram:

a) custos associados a sistemas híbridos: incluem o custo com investimento inicial, o custo para reposição dos equipamentos e custos referentes à operação e à manutenção (O\&M);

b) disponibilidade dos recursos energéticos: são fornecidos os dados de irradiância solar e velocidade média anual do vento da região na qual o projeto será realizado;

c) demanda solicitada pela carga: a demanda de energia elétrica que o sistema deve atender em um determinado intervalo de tempo;

d) potências e quantidades dos componentes: valores utilizados na simulação de vários cenários de otimização a fim de identificar a melhor configuração do sistema.

\subsection{Características do sistema de microrrede desenvolvido}

O projeto de microrrede apresentado foi dimensionado tomando como base um condomínio residencial contendo trinta e seis residências. A escolha desse tipo de carga foi baseada no alto consumo de energia elétrica apresentado por condomínios.

O primeiro passo para o dimensionamento de uma microrrede é determinar a demanda de energia necessária para atender a carga. Inicialmente, determinou-se o número de pessoas em cada residência por meio de sorteio aleatório, obtido através de um programa 
desenvolvido no MATLAB. Segundo dados do IBGE, a média de moradores por domicílio para a região Sudeste é de 2,92 (INSTITUTO BRASILEIRO DE GEOGRAFIA E ESTATÍSTICA, 2015). Portanto, o sorteio foi realizado considerando um intervalo entre 2 e 6 moradores para que o resultado final fosse o mais balanceado possível. O número total de pessoas residentes no condomínio foi de 130. Considerou-se também que o consumo de energia elétrica por pessoa era de aproximadamente $653 \mathrm{kWh} / \mathrm{ano}$, de acordo com World Energy Council (2016). Conhecendo o número de moradores do condomínio e o consumo de energia elétrica por pessoa, foi possível estimar a demanda mensal total. Mas, antes, foram feitas as seguintes considerações:

a) aumento de carga no condomínio futuramente: considerando que nos próximos anos a carga do condomínio possa aumentar, devido a novos equipamentos adquiridos ou pela chegada de novos moradores, o sistema será sobredimensionado em 15\%;

b) perdas devido à utilização de baterias: os sistemas de armazenamento que utilizam bancos de baterias apresentam um percentual de perdas de aproximadamente $35 \%$. Esse valor deve ser considerado no dimensionamento do sistema.

Considerando os fatores mencionados, a demanda final foi de 11,07 MWh/mês. O próximo passo foi dimensionar o sistema contendo as fontes renováveis. Após a análise de alguns fatores, como irradiância solar e velocidade média do vento, para a cidade de Janaúba/MG, concluiu-se que esta possui potencial eólico superior ao solar. Com isso, por decisão do projetista, o sistema eólico será responsável pelo fornecimento de $65 \%$ da demanda total, resultando em uma geração de 7,20 MWh, e os 35\% restantes serão fornecidos pelo sistema fotovoltaico, correspondente a 3,87 MWh mensais.

\subsubsection{Dimensionamento do sistema eólico}

A Tabela 1mostra a velocidade média trimestral do vento para a cidade de Janaúba/MG a uma altura de $50 \mathrm{~m}$. A fim de garantir que haja energia disponível também nos meses de menor recurso, a velocidade média utilizada nos cálculos foi de $5,9 \mathrm{~m} / \mathrm{s}$, valor registrado nos meses de dezembro, janeiro e fevereiro. 
Tabela 1 - Velocidade média do vento para uma altura de $50 \mathrm{~m}$.

\begin{tabular}{ccccc}
\hline \multicolumn{5}{c}{ Velocidade média do vento a 50 m de altura $(\mathbf{m} / \mathbf{s})$} \\
\cline { 1 - 2 } Dez-Fev & Mar-Mai & Jun-Ago & Set-Nov & Anual \\
5,9 & 6,34 & 8,03 & 7,33 & 6,9 \\
\hline
\end{tabular}

Fonte: Adaptado de Cresesb Eólica (2013).

A turbina eólica escolhida para dimensionamento do sistema é da marca Whisper. A escolha foi baseada na baixa velocidade inicial do vento necessária para que ela entre em operação, resultando em uma produção maior e diminuindo riscos de indisponibilidade de energia.

Para um valor de velocidade média do vento a $5,9 \mathrm{~m} / \mathrm{s}$, tem-se que a energia gerada por uma turbina é de $649 \mathrm{kWh} / \mathrm{mês}$. Considerando que a potência total gerada pelo sistema eólico deve ser de 7,2 MWh/mês, são necessárias 11 turbinas eólicas.

\subsubsection{Dimensionamento do sistema fotovoltaico}

Os valores mensais da irradiância solar para a cidade de Janaúba são mostrados na Tabela 2,os quais são obtidos para uma inclinação dos painéis solares de $15^{\circ}$, sendo que essa angulação resulta na maior média anual da irradiância solar.

Tabela 2 - Irradiação solar diária média para a cidade de Janaúba/MG.

\begin{tabular}{ccccccccccccc}
\hline \multicolumn{10}{c}{ Irradiação solar diária média $\left(\mathbf{k W h} / \mathbf{m}^{2}\right.$.dia) } \\
\hline Jan & Fev & Mar & Abr & Maio & Jun & Jul & Ago & Set & Out & Nov & Dez \\
5,33 & 5,42 & 4,58 & 4,64 & 4,69 & 4,19 & 4,58 & 5,56 & 5,19 & 5,75 & 5,28 & 5,56 \\
\hline
\end{tabular}

Fonte: Adaptado de Cresesb Eólica (2013).

O módulo fotovoltaico utilizado no dimensionamento é da marca SunPower, modelo SPR-440NE-WHT-D, com potência nominal de $440 \mathrm{~W}$. Como pode ser visto na Tabela 2, o mês de março apresentou o menor valor registrado de irradiância solar quando comparado aos outros meses, sendo de 4,61 kWh/m².dia. Portanto, tem-se cerca de 4,61 horas de sol pleno para o mês mais crítico do ano.O cálculo da energia produzida pelo módulo pode ser realizado pela Equação (1) (VILLALVA; GAZOLI, 2012): 


$$
E_{p}=E_{S} A_{M} \eta_{M}
$$

onde $\mathrm{E}_{\mathrm{p}}$ é a energia produzida pelo módulo diariamente em Wh; Es é a irradiância diária em $\mathrm{Wh} / \mathrm{m}^{2} /$ dia; $\mathrm{A}_{\mathrm{M}}$ é a área da superfície do módulo em $\mathrm{m}^{2}$; e $\eta_{M}$ é a eficiência do módulo.

Considerando os valores de área e eficiência do módulo como sendo $2,16 \mathrm{~m}^{2} \mathrm{e}$ 21,3\%, respectivamente, e a irradiância mínima registrada de $4,61 \mathrm{kWh} / \mathrm{m}^{2}$.dia, a energia produzida mensalmente por um módulo são 63,63 kWh. Assim, são necessários 60 módulos para se obter uma geração mensal de energia de 3,87 MWh.

Com a apresentação da metodologia desenvolvida para o dimensionamento da microrrede eólica e solar, na próxima seção são apresentados os resultados e as discussões do sistema implementado neste artigo.

\section{RESULTADOS}

Após a inserção dos dados de entrada no HOMER, o programa foi simulado e encontrou-se uma solução ideal para a microrrede. Por meio das simulações realizadas, foi possível verificar que uma solução é apontada apenas quando o sistema consegue suprir a demanda especificada. A Figura 7 mostra a melhor solução encontrada pelo programa para o sistema proposto.

\begin{tabular}{|c|c|c|c|c|c|c|c|c|c|c|}
\hline$\triangle$ & 紧个 & $\tilde{z}$ & ${ }_{(\mathrm{kW})}^{\mathrm{PV}} \mathrm{P}$ & G3 8 & $1 \mathrm{kWh}$ LA $\checkmark$ & $\begin{array}{c}\text { Converter } \nabla \\
(\mathrm{kW})\end{array}$ & $\begin{array}{l}\mathrm{COE} \\
(\$)\end{array}$ & ${ }_{(\$)}^{N P C} \nabla$ & $\begin{array}{c}\text { Operating cost } \nabla \\
\text { (\$) }\end{array}$ & $\begin{array}{c}\text { Initial capital } \\
\text { (\$) }\end{array}$ \\
\hline & 羂 & $5 \%$ & 28.0 & 9 & 300 & 100 & $\$ 1.42$ & $\$ 2.07 \mathrm{M}$ & $\$ 101,685$ & $\$ 760,229$ \\
\hline
\end{tabular}

Figura 7- Solução ideal calculada pelo HOMER. Fonte: Fonte: Homer Energy (2016).

O programa sugere que sejam utilizadas 9 turbinas eólicas (“G3”) para o sistema eólico ao invés de 11, como calculado na seção 3.2.1. Isso pode ser explicado pelo fato de que, no cálculo feito para determinar o número de turbinas eólicas, a velocidade média do vento considerada é a menor registrada no ano. Entretanto, o programa considera as demais velocidades médias mensais que apresentam valores superiores e, consequentemente, maior geração. O número de baterias ("1 kWh LA") ideal é de 300, resultando em uma autonomia 
da microrrede de aproximadamente 12 horas, ou seja, caso haja alguma falha no sistema e a geração seja comprometida, o banco de baterias será capaz de fornecer energia para a carga por um período de até 12 horas. O conversor ("Converter") possui potência de $100 \mathrm{~kW}$, valor máximo que o sistema Ingecon Hybrid MS suporta. O custo médio da energia gerada (“COE”) é de $\$ 1,42$ por $\mathrm{kWh}$, e o sistema é capaz de fornecer $143.798 \mathrm{kWh} / \mathrm{ano}$, referente a uma geração diária de 399,44 kWh. Sendo assim, pode-se concluir que a microrrede é capaz de suprir a demanda do condomínio (369 kWh/dia).

O sistema eólico é responsável por 66,5\% da energia total gerada, enquanto o sistema fotovoltaico fornece $33,5 \%$. Tal informação pode ser comprovada pela Figura 8, que mostra a produção de energia mensal referente a cada fonte, eólica e solar. Cada coluna representa a produção mensal total dos dois sistemas, sendo que a coluna verde indica a geração do sistema eólico, e a coluna laranja, a geração do sistema solar.

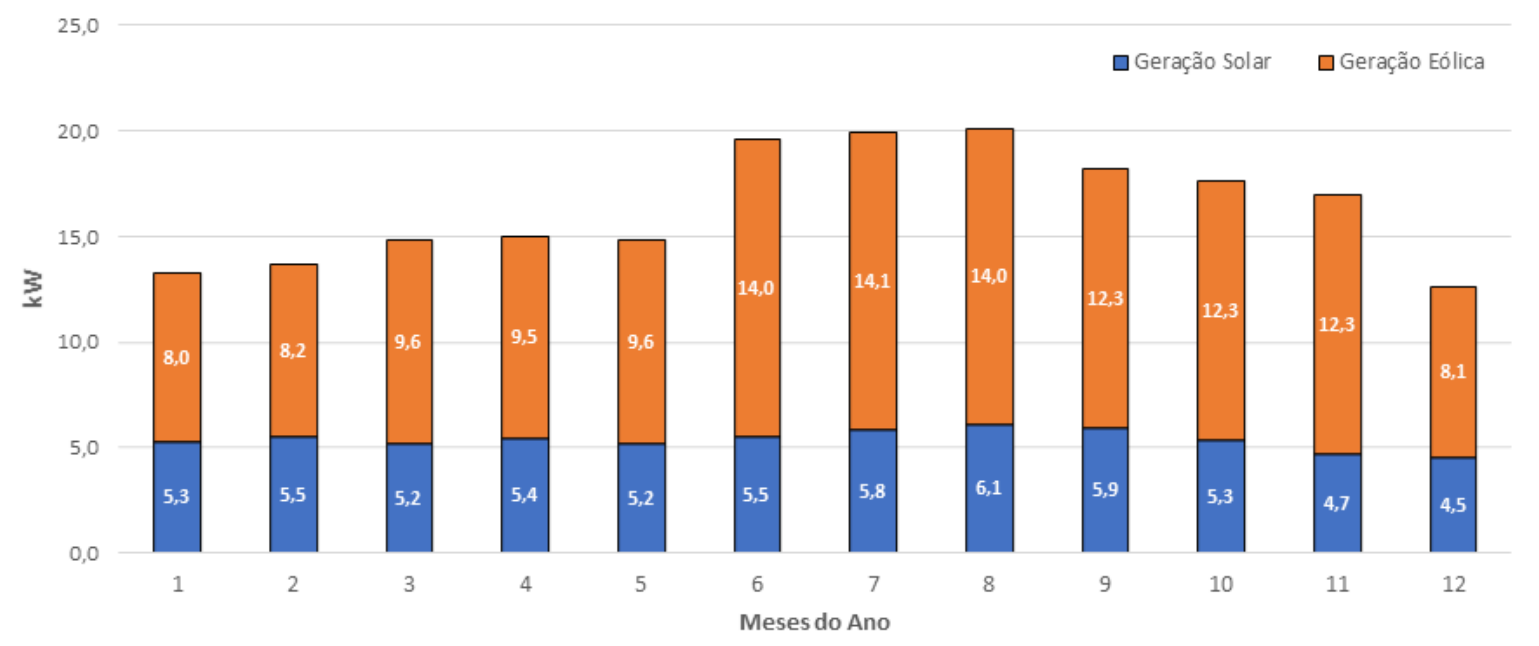

(a)

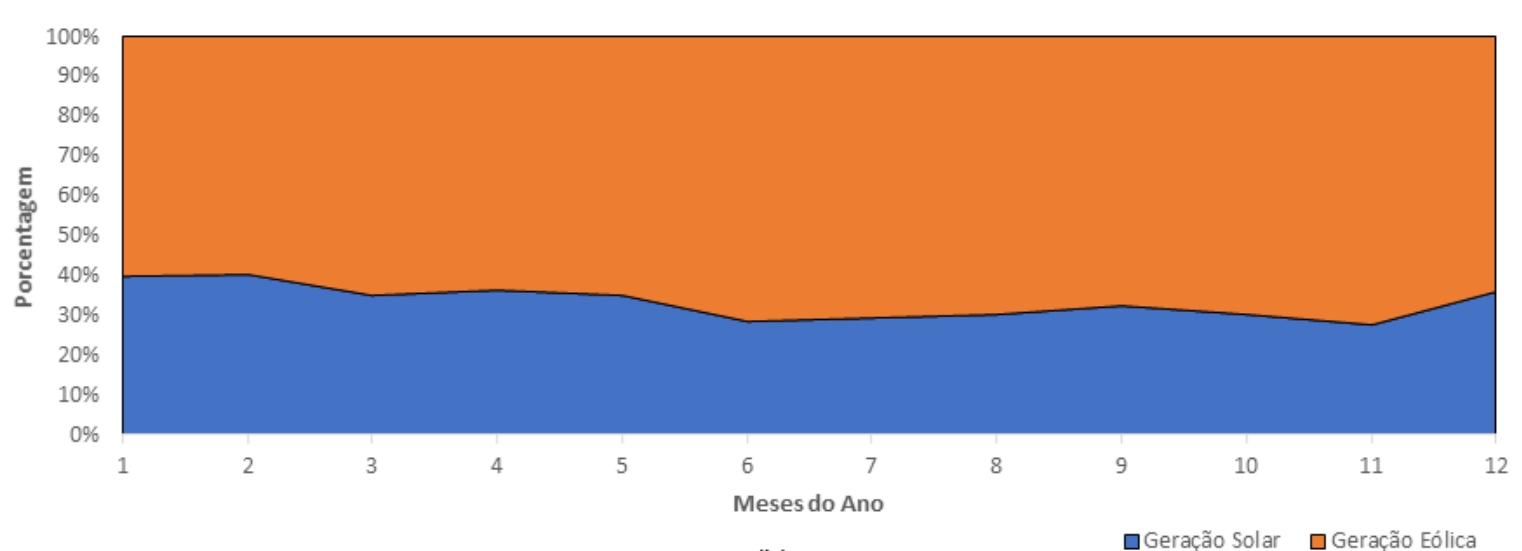

(b)

Figura 8 - Relação da geração de energia dos sistemas eólico e solar Fonte: Fonte: Homer Energy (2016). (a) Produção média mensal (b) Porcentagem de energia obtida pelas gerações solar e eólica, valores obtidos através da simulação no software HOMER.

ForSci.: r. cient. IFMG, Formiga, v. 5, n. 3, e00283, jul./dez. 2017. 
Como pode ser visto na Figura 9, a microrrede apresenta um excedente de energia produzido anualmente de $11,2 \%$. Com isso, além do sistema ser capaz de suprir a demanda do condomínio, ele possibilita que os consumidores forneçam energia elétrica para a rede local, gerando créditos com a concessionária que podem ser utilizados posteriormente. Neste caso, se houver necessidade de abastecimento por meio da distribuidora local, o valor das faturas de energia elétrica pode ser reduzido por meio desses créditos, de acordo com a Resolução Normativa nº87, da ANEEL, de 2015 (AGÊNCIA NACIONAL DE ENERGIA ELÉTRICA, 2015).

\begin{tabular}{|c|c|c|c|c|c|c|}
\hline Production & kWh/yr & $\%$ & \multicolumn{2}{|c|}{ Consumption } & $\mathrm{kWh} / \mathrm{yr}$ & $\%$ \\
\hline Generic flat plate PV & 48,167 & 33.50 & \multicolumn{2}{|c|}{ AC Primary Load } & 113,006 & 100.00 \\
\hline Generic 3 kW & 95,631 & 66.50 & \multirow{2}{*}{\multicolumn{2}{|c|}{$\begin{array}{l}\text { DC Primary Load } \\
\text { Total }\end{array}$}} & 0 & 0.00 \\
\hline Total & 143,798 & 100.00 & & & 113,006 & 100.00 \\
\hline & \multicolumn{2}{|c|}{ Quantity } & $\mathrm{kWh} / \mathrm{yr}$ & $\%$ & & \\
\hline & \multicolumn{2}{|c|}{ Excess Electricity } & $16,037.6$ & 11.2 & & \\
\hline & \multicolumn{2}{|c|}{ Unmet Electric Load } & $21,679.0$ & 16.1 & & \\
\hline & \multicolumn{2}{|c|}{ Capacity Shortage } & $30,582.2$ & 22.7 & & \\
\hline
\end{tabular}

Figura 9 - Geração elétrica obtida pela microrrede. Fonte: Fonte: Homer Energy (2016).

A capacidade do sistema fotovoltaico é de $28 \mathrm{~kW}$, e o maior valor de potência obtido na saída é de 26,8 kW. O sistema produz energia durante 4.406 horas/ano, o que equivale a aproximadamente 12 horas de operação por dia. A geração diária é de 131,96 kWh, o que representa $35 \%$ da demanda solicitada. Apesar do elevado número de horas de funcionamento do sistema fotovoltaico, ele não apresenta um nível satisfatório de geração. Isso acontece porque, a irradiância solar registrada na cidade de Janaúba/MG, em determinados períodos, não apresenta índices elevados. A Figura 10 mostra os resultados obtidos na simulação para o sistema fotovoltaico. 


\begin{tabular}{|l|l|l|}
\hline Quantity & Value & Units \\
\hline Rated Capacity & 28.00 & $\mathrm{~kW}$ \\
\cline { 1 - 2 } Mean Output & 5.50 & $\mathrm{~kW}$ \\
Mean Output & 131.96 & $\mathrm{kWh} / \mathrm{d}$ \\
Capacity Factor & 19.64 & $\%$ \\
Total Production & $48,166.86$ & $\mathrm{kWh} / \mathrm{yr}$ \\
\hline
\end{tabular}

\begin{tabular}{|l|l|l|}
\hline Quantity & Value & Units \\
\hline Minimum Output & 0.00 & $\mathrm{~kW}$ \\
Maximum Output & 26.8 & $\mathrm{~kW}$ \\
PV Penetration & 35.8 & $\%$ \\
Hours of Operation & 4,406 & $\mathrm{hrs} / \mathrm{yr}$ \\
Levelized Cost & 0.167 & $\$ / \mathrm{kWh}$ \\
\hline
\end{tabular}

Figura 10 - Desempenho do sistema fotovoltaico. Fonte: Fonte: Homer Energy (2016).

A potência instalada do sistema eólico é de $27 \mathrm{~kW}$, fornecendo o equivalente a 265,64 $\mathrm{kWh} /$ dia. Com isso, este sistema é capaz de fornecer até $72 \%$ da energia utilizada no condomínio diariamente. Como a geração fotovoltaica fica comprometida em determinados períodos, o sistema eólico é capaz de atender grande parte da demanda durante estes intervalos. Tais informações podem ser confirmadas pela Figura 11.

\begin{tabular}{|l|l|l|}
\hline Quantity & Value & Units \\
\hline Total Rated Capacity & 27.00 & $\mathrm{~kW}$ \\
Mean Output & 10.92 & $\mathrm{~kW}$ \\
Capacity Factor & 40.43 & $\%$ \\
Total Production & $95,631.17$ & $\mathrm{kWh} / \mathrm{yr}$ \\
\hline
\end{tabular}

\begin{tabular}{|l|l|l|}
\hline Quantity & Value & Units \\
\hline Minimum Output & 0.00 & $\mathrm{~kW}$ \\
Maximum Output & 32.40 & $\mathrm{~kW}$ \\
Wind Penetration & 71.00 & $\%$ \\
Hours of Operation & $7,607.00$ & $\mathrm{hrs} / \mathrm{yr}$ \\
Levelized Cost & 0.13 & $\mathrm{~S} / \mathrm{kWh}$ \\
\hline
\end{tabular}

Figura 11- Desempenho do sistema eólico.

Fonte: Fonte: Homer Energy (2016).

Os resultados mostram que o sistema Ingecon Hybrid MSutilizadoé capaz de suportar a energia gerada pelo sistema, pois, de acordo com seus dados técnicos, o componente suporta até $45 \mathrm{~kW}$ de geração fotovoltaica e $45 \mathrm{~kW}$ de geração eólica. Como pode ser visto na Figura 12, a energia que entra no inversor é de 49.045,95 kWh/ano, enquanto na saída tem-se 44.141,36 kWh/ano. Portanto, o total de perdas no inversor é de $10 \%$. 

geração conjunta eólica/solar aplicada em um condomínio residencial na cidade de Janaúba / MG.

\begin{tabular}{|l|l|l|l|}
\hline Quantity & Inverter & Rectifier & Units \\
\hline Capacity & 100.00 & 90.00 & $\mathrm{~kW}$ \\
\cline { 1 - 3 } Mean Output & 5.04 & 1.63 & $\mathrm{~kW}$ \\
Minimum Output & 0.00 & 0.00 & $\mathrm{~kW}$ \\
Maximum Output & 39.55 & 23.55 & $\mathrm{~kW}$ \\
Capacity Factor & 5.04 & 1.63 & $\%$ \\
\hline
\end{tabular}

\begin{tabular}{|l|l|l|l|}
\hline Quantity & Inverter & Rectifier & Units \\
\hline Hours of Operation & $5,462.00$ & $2,191.00$ & $\mathrm{hrs} / \mathrm{yr}$ \\
Energy Out & $44,141.36$ & $14,301.99$ & $\mathrm{kWh} / \mathrm{yr}$ \\
Energy In & $49,045.95$ & $16,825.87$ & $\mathrm{kWh} / \mathrm{yr}$ \\
Losses & $4,904.60$ & $2,523.88$ & $\mathrm{kWh} / \mathrm{yr}$ \\
\hline
\end{tabular}

Figura 12 - Resultados obtidos para o inversor. Fonte: Fonte: Homer Energy (2016).

A utilização do sistema de armazenamento contendo baterias é importante para que a confiabilidade no fornecimento de energia seja aumentada. Sendo assim, quando a geração proveniente das fontes renováveis não for suficiente para atender a demanda, as baterias serão utilizadas. Quanto maior o número de baterias, maior será a autonomia do sistema e, consequentemente, o custo se tornará mais elevado. Como mostra a Figura 13, utilizando-se um total de 300 baterias, tem-se uma autonomia de quase 12 horas. Além disso, a energia recebida pelas baterias foi de 37.435,61 $\mathrm{kWh} / \mathrm{ano}$, e a fornecida a carga foi $30.109,61$ $\mathrm{kWh} /$ ano. Portanto, o total de perdas obtido no sistema de armazenamento foi de $19 \%$.

\begin{tabular}{|c|c|c|c|c|c|c|}
\hline Quantity & Value & Units & \multicolumn{2}{|c|}{ Quantity } & Value & Units \\
\hline Autonomy & 11.72 & $\mathrm{hr}$ & \multicolumn{2}{|c|}{ Average Energy Cost } & 0.00 & $\$ / \mathrm{kWh}$ \\
\hline Storage Wear Cost & 3.54 & $\$ / \mathrm{kWh}$ & \multicolumn{2}{|c|}{ Energy In } & $37,435.61$ & $\mathrm{kWh} / \mathrm{yr}$ \\
\hline Nominal Capacity & 300.24 & $\mathrm{kWh}$ & \multicolumn{2}{|c|}{ Energy Out } & $30,109.61$ & $\mathrm{kWh} / \mathrm{yr}$ \\
\hline Usable Nominal Capacity & 180.14 & $\mathrm{kWh}$ & \multicolumn{2}{|c|}{ Storage Depletion } & 180.14 & $\mathrm{kWh} / \mathrm{yr}$ \\
\hline Lifetime Throughput & $141,960.00$ & $\mathrm{kWh}$ & \multicolumn{2}{|c|}{ Losses } & $7,145.85$ & $\mathrm{kWh} / \mathrm{yr}$ \\
\hline Expected Life & 4.22 & $\mathrm{yr}$ & \multicolumn{2}{|c|}{ Annual Throughput } & $33,663.57$ & $\mathrm{kWh} / \mathrm{yr}$ \\
\hline & \multicolumn{2}{|c|}{ Quantity } & Value & Units & & \\
\hline & \multicolumn{2}{|c|}{ Batteries } & \multicolumn{2}{|l|}{300.00} & & \\
\hline & \multicolumn{2}{|c|}{ String Size } & \multicolumn{2}{|l|}{20.00} & & \\
\hline & \multicolumn{2}{|c|}{ Strings in Parallel } & \multicolumn{2}{|l|}{15.00} & & \\
\hline & \multicolumn{2}{|c|}{ Bus Voltage } & \multicolumn{2}{|c|}{240.00} & & \\
\hline
\end{tabular}

Figura 13 - Resultados obtidos para o banco de baterias Fonte: Homer Energy (2016). 


\subsection{Análise econômica do projeto}

Uma vez finalizado o dimensionamento da microrrede e conhecendo os principais componentes do sistema, é possível estimar o investimento que este tipo de projeto requer. A Tabela 3 mostra a quantidade dos equipamentos utilizados e seus respectivos preços. Não foi disponibilizado pelo fabricante o valor do sistema Ingecon Hybrid MS, portanto, para se obter um valor final adequado, utilizou-se o preço de um equipamento com funcionalidade similar, o inversor Kaco.

Tabela 3 - Quantidade e custo estimado dos equipamentos da microrrede

\begin{tabular}{clclll}
\hline Item & \multicolumn{1}{c}{ Descrição } & Qtde & Preço unitário (R\$) & Preço total (R\$) \\
\hline 1 & $\begin{array}{l}\text { Turbinaeólica Whisper } \\
\text { Southwest Windpower } \\
\text { Kit torre + cabos + acessórios } \\
\text { para turbina eólica }\end{array}$ & 9 & $\mathrm{R} \$ 24.869,25$ & $\mathrm{R} \$ 223.823,25$ \\
2 & $\mathrm{R} \$ 2.831,85$ & $\mathrm{R} \$ 25.486,65$ \\
3 & $\begin{array}{l}\text { Painel solar modelo SPR- } \\
\text { 440NE-WHT-D, Sunpower }\end{array}$ & 60 & $\mathrm{R} \$ 1.500,00$ & $\mathrm{R} \$ 90.000,00$ \\
4 & $\begin{array}{l}\text { Suporte de alumínio para } \\
\text { módulos solares }\end{array}$ & 4 & $\mathrm{R} \$ 2.579,29$ & $\mathrm{R} \$ 10.317,60$ \\
5 & $\begin{array}{l}\text { Baterias 105 Ah, Moura } \\
\text { Inversor Kaco 50 kW }\end{array}$ & 300 & $\mathrm{R} \$ 550,00$ & $\mathrm{R} \$ 165.000,00$ \\
6 & 1 & $\mathrm{R} \$ 28.800,00$ & $\mathrm{R} \$ 28.800,00$ \\
\hline
\end{tabular}

Como pode ser visto na Tabela 3, o preço total do projeto é de aproximadamente $\mathrm{R} \$$ 543.427,50, sendo que este levantamento econômico não levou em conta o custo da mão de obra e a documentação para execução do projeto proposto. Considerando ainda que o condomínio possui um total de trinta e seis residências, pode-se estimar o custo por residência em torno de $\mathrm{R} \$ 15.095,20$.

A fim de estimar o tempo de retorno de investimento (payback simples) do projeto, realizou-se o cálculo aproximado de quanto o condomínio pagaria de faturamento de energia elétrica caso a demanda necessária fosse contratada junto à CEMIG (Companhia Energética de Minas Gerais). Para o caso de condomínios residenciais, a estrutura tarifária convencional é a mais indicada, considerando que o período de maior utilização de energia elétrica ocorre entre $18 \mathrm{~h}$ e $21 \mathrm{~h}$. Utilizando esse tipo de tarifação, o faturamento é feito levando em conta 
tanto a demanda contratada quanto o consumo (COMPANHIA ENERGÉTICA DE MINAS GERAIS, 2011).

De acordo com tarifas da estrutura convencional fornecidas pela CEMIG, referentes ao mês de outubro de 2016, a tarifa relativa à demanda é de $\mathrm{R} \$ 42,29 / \mathrm{kW}$ enquanto a de consumo é de R\$446,09/MWh, incluindo todos os impostos aplicáveis (COMPANHIA ENERGÉTICA DE MINAS GERAIS, [2016]). Assim, é possível estimar que o condomínio pagaria aproximadamente uma quantia mensal de $\mathrm{R} \$ 7.264,38$. Com isso, tem-se uma estimativa do valor de tempo de retorno de investimento (payback simples) de sete anos, para os custos dos equipamentos obtidos por meio do dimensionamento proposto.

Levando em consideração todos os benefícios envolvidos na utilização das fontes renováveis em sistemas de geração, o período de tempo estimado é relativamente baixo, uma vez que o sistema contribui para uma geração de energia de forma mais limpa, além de apresentar alta confiabilidade.

\section{CONCLUSÃO}

O objetivo principal deste trabalho foi dimensionar e simular uma microrrede, sistema considerado uma aplicação da geração distribuída. A simulação foi feita através do software HOMER, desenvolvido especificamente para a análise de microrredes. O projeto foi realizado com aplicação para um condomínio contendo um total de trinta e seis residências, na cidade de Janaúba/MG.

Por meio da simulação, pôde-se perceber que os dados da topologia considerada ideal para a microrrede foram semelhantes aos valores previamente calculados, ressaltando ainda que o sistema projetado possui autonomia de quase 12 horas, aumentando assim a sua confiabilidade.

Os resultados indicaram um excedente na produção de energia de $11,2 \%$ ao ano. Considerando que a microrrede pode operar paralelamente à rede elétrica convencional, esse excedente pode ser injetado na rede e gerar créditos para os condôminos, diminuindo o faturamento final de energia, de acordo com a Resolução Normativa da ANEEL n ${ }^{\circ} 687$.

Portanto, conclui-se que o sistema analisado pode ser considerado eficaz, pois é possível suprir a demanda de energia elétrica necessária para atender o condomínio, oferecendo confiabilidade no abastecimento devido a sua autonomia. Além disso, a utilização 
de uma microrrede na cidade de Janaúba/MG contribui para uma geração mais limpa de energia, aproveitando o potencial disponível das fontes renováveis.

\title{
DIMENSIONING AND SIMULATION OF A MICRO-NETWORK USING SOLAR/WIND GENERATION APPLIED IN A RESIDENTIAL CONDOMINIUM IN THE CITY OF JANAÚBA/MG
}

\begin{abstract}
A micro-network can be defined as a small power generation system, containing distributed generating units that usually use different power sources, to support a certain number of consumer units. In Brazil, some factors have contributed to a greater insertion of micronetwork in the electrical system emphasizing the current water crisis, the existence of consumption centers away from the transmission and/or distribution system, environmental issues and high potential of renewable sources in the country. Thus, the objective of this work is to scale out and simulate a micro-network using wind / solar generation, together with an energy storage system, to meet the demand of a condominium with thirty-six residences, in the city of Janaúba/MG, which has a high potential for wind and solar generation. The simulation of the project was carried out through the software HOMER, which is an open source computer tool, specialized in the analysis of micro-network. Given the values of solar irradiance and wind speed in the city of Janaúba/MG, it was possible to conclude that the region has higher wind potential than solar. Thus, the system was satisfactorily sized $66.5 \%$ of its total demand supplied by the wind system and the remaining $33.5 \%$ by the solar system.
\end{abstract}

Keywords: Micro-network. Renewable sources. Software HOMER.

\section{REFERÊNCIAS}

AGÊNCIA NACIONAL DE ENERGIA ELÉTRICA. Resolução Normativa nº 687, de 24 de novembro de 2015. Diário Oficial da União, n. 230, seção 1, p. 45, 2 dez. 2015. Disponível em: <http://www.lex.com.br/legis_27047031_RESOLUCAO_NORMATIVA_N_687_DE_24 _DE_NOVEMBRO_DE_2015.aspx>. Acesso em 12 set. 2016.

Micro e minigeração distribuída: sistema de compensação de energia elétrica. Brasília, 2014.

COMPANHIA ENERGÉTICA DE MINAS GERAIS. Atlas eólico Minas Gerais. Belo Horizonte, 2010.

Atlas Solarimétrico de Minas Gerais. Belo Horizonte, 2012. 
Manual de Gerenciamento de Energia. Belo Horizonte, 2011.

Valores de tarifas e serviços. Belo Horizonte, [2016]. Disponível em: $\langle$ https://www.cemig.com.br/ptr/atendimento/Paginas/valores_de_tarifa_e_servicos.aspx >. Acesso em: 28 out. 2016.

CRESESB EÓLICA. Potencial eólico: atlas do potencial eólico brasileiro. Brasília, 2013. Disponível em: <http://www.cresesb.cepel.br/index.php\#data>. Acesso em: 1 set. 2016.

CRUZ, J. L. C.Geração distribuída.O Setor Elétrico, n. 93, p. 70-76, out. 2013.

GOMES, A. Matriz cada vez mais diversificada. O Setor Elétrico, n. 95, p. 52-59, dez. 2013.

INSTITUTO BRASILEIRO DE GEOGRAFIA E ESTATÍSTICA. Síntese de indicadores sociais: uma análise das condições de vida da população brasileira. Rio de Janeiro, 2015.

INGETEAM. Solar energy solution: product catalog 2013. Zamudio: INGECON, 2013. Disponível em: <http://ingeteam.it/userfiles/allegati/ingeconsun_catalogo_en_bassa_ 1365412359.pdf>. Acesso em: 09 de setembro de 2016.

NATIONAL RENEWABLE ENERGY LABORATORY. Getting started guide for HOMER legacy. Colorado, 2011.

HOMER ENERGY. Homer pro: software for simulation, optimization and sensitivity analysis. Versão 3.7. Boulder, 2016. Disponível em: <http://www.homerenergy.com/HOMER _pro.html>. Acesso em: 12 de setembro de 2016.

OPENEI. HOMER. Disponível em: <http://en.openei.org/wiki/HOMER>. Acesso em: 12 set. 2016.

OUDALOV, A.; BUEHLER, T.; CHARTOUNI, D. Utility scale applications of energy storage. Atlanta: IEEE Energy Conference, 2008.

PASSOS, R. S. D. Estudo de caso do impacto da microgeração na tensão e nas perdas de um sistema de distribuição. 2014. 69 f. Monografia (Trabalho de conclusão de graduação) Departamento de Engenharia Elétrica, Universidade Federal do Rio Grande do Sul, Porto Alegre, 2014.

RAYTHEON COMPANY. How to build a power base. Waltham, 2016. Disponível em: <http://www.raytheon.com/news/feature/power_tech.html>. Acesso em: 11 de maio de 2016. 
SILVA, F. L. D. Análise do desempenho de uma microrrede com múltiplas unidades geradoras. 2011. 103 f. Dissertação (Mestrado em Engenharia Elétrica) - Universidade Federal do Espírito Santo, Vitória, 2011.

SINGH, T. C. et al. Design features of energy storage systems for Green buildings: an economic comparison with off-grid and on-grid solutions. International Research Journal of Engineering and Technology, v. 3, n. 5, p. 38-42, maio 2016.

SU, W.; WANG, J. Energy management systems in microgrid operations.The Electricity Journal, v. 25, n. 8, p. 45-60, out. 2012.

VILLALVA, M. G.; GAZOLI, J. R. Energia Solar Fotovoltaica: conceitos e aplicações: sistemas isolados e conectados à rede. São Paulo: Érica, 2012.

WORLD ENERGY COUNCIL. Average electricity comsumption of households per capita. Londres, 2016. Disponível em: <https://www.wec-indicators.enerdata.eu/electricityuse-per- capita.html>. Acesso em: 1 set. de 2016.

Recebido em: 31/07/2017

Aprovado em: 08/08/2017

Publicado em: 13/11/2017 